\title{
Antitumour responses with more BiTE
}

To date, immunotherapeutic approaches that target intracellular tumour antigens are mostly limited to complex cellular therapies. Now, reporting in Nature Biotechnology, Scheinberg and colleagues present a novel bispecific $\mathrm{T}$ cell engager (BiTE) construct that combines the $\mathrm{T}$ cell receptor (TCR)-like recognition of intracellular tumour antigens with the pharmacologically attractive features of monoclonal antibodies. This construct induced potent cytotoxic $\mathrm{T}$ cell (CTL) mediated antitumour responses in a number of different cancer models.

BiTEs are heterodimers of immunoglobulin $\mathrm{G}$ single chains, of which one chain is specific for a defined tumour-associated antigen,

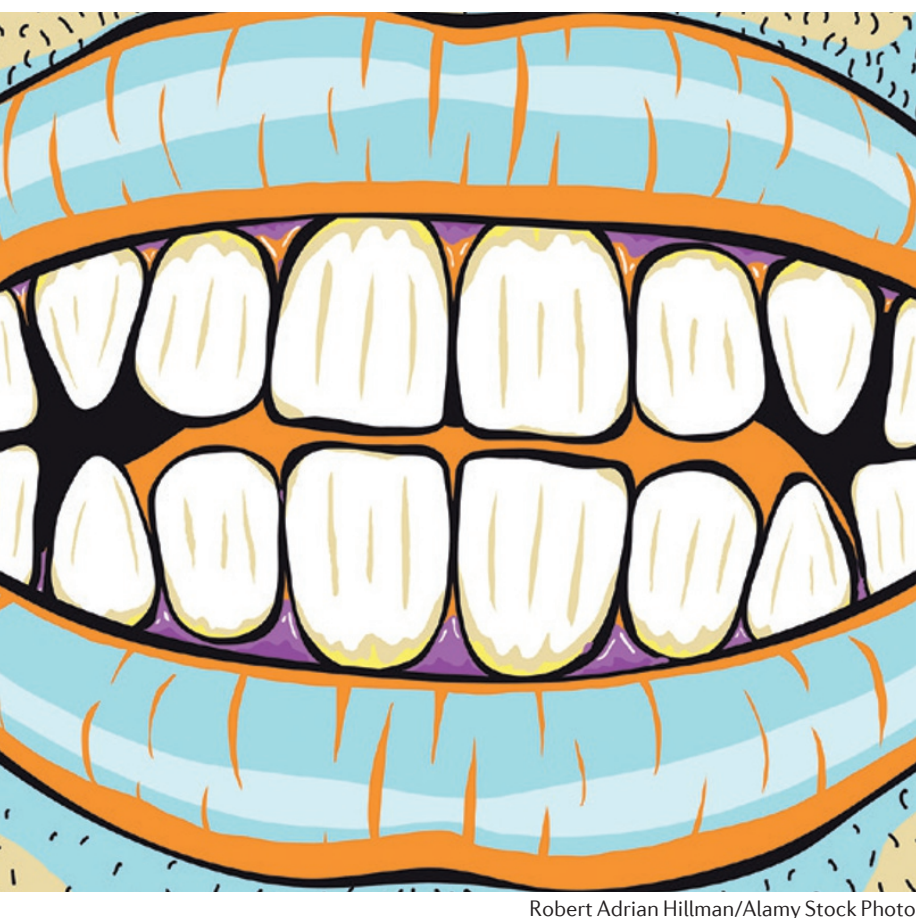

and the other for CD3 (the TCR complex). Such constructs can redirect $\mathrm{T}$ cells to cancer cells and induce cell killing, independent of the $T$ cell's intrinsic antigen-specific TCR recognition. A first BiTE construct, directed at the high-density antigen CD19 (a B-cell specific antigen), has been approved for the treatment of B cell neoplasms.

Here, the authors based their BiTE on the TCR-mimic antibody ESK1, which binds to a peptide derived from Wilms tumour protein 1 (WT1), an oncofetal protein that is expressed at a low density on many different tumour cells. ESK1-BiTE (at concentrations as low as $3 \mathrm{ng}$ per $\mathrm{ml}$ ) was shown to facilitate CTL-mediated killing of several ESK1-reactive cancer cell lines. These effects were also confirmed in an autologous setting, using peripheral blood mononuclear cells (PBMCs) and tumour cells from a patient with ovarian cancer.

Next, BiTE activity was assessed in mouse xenograft models. To prevent non-cancer specific graft rejection, xenografts are usually carried out in mice lacking $\mathrm{T}$ or B cells. In order to assess BiTE-mediated $\mathrm{T}$ cell activation in these models, the authors generated Epstein Barr virus (EBV)-specific human $\mathrm{T}$ cells and injected them into the mice 4 days after engraftment of the tumour cells. T cells and tumour cells were tagged with different luminescent tags, which allowed kinetic measurements in living mice. Mice injected with the aggressive acute myeloid leukaemia SET-2 cell line usually succumb to leukaemia within one month. By contrast, mice that received intravenuous BiTEs 6 hours after the $\mathrm{T}$ cell transfer and then daily for 6 days showed no evidence of disease at day 14 , and only a minimal disease burden up to day 25. Similar results were seen in mice injected with primary acute lymphoblastic leukaemia cells or with mesothelioma cells, which represent an aggressive solid tumour.

The mechanism of BiTE activity has been exclusively attributed to the capacity of the construct to bridge cancer cell targets and $\mathrm{T}$ cells to form a 'cytolytic synapse.' To investigate whether this process can also activate T cells in the polyclonal population that have a pre-existing specificity for the cancer cells, the authors co-cultured PBMCs with autologous tumour cells from ovarian cancer patients in the presence of low-dose ESK1-BiTE. Indeed, a strong secondary response against the cancer antigen HER2 and other undefined cancer antigens was detected (a phenomenon called 'epitope spreading'). The ESK1-BiTE-mediated interaction between tumour cells and T cells was strictly required for both primary and secondary responses.

Although difficult to model in animals, the authors speculate that epitope spreading could provide broad, effective long-term responses, as it prevents the outgrowth of tumour cells that have downregulated or do not express the initially targeted antigen.

Alexandra Flemming

ORIGINAL RESEARCH PAPER Dao, T. et al.

Therapeutic bispecific T-cell engager antibody targeting the intracellular oncoprotein WT1. Nat. Biotechnol. 33, 1079-1086 (2015) 